\title{
Human Hair Catalyzed Selective Reduction of Nitroarenes to
}

\author{
Amines \\ Mohammad Gholinejad *a,b, Hamid Esmailoghli, ${ }^{\text {a }}{ }^{\text {José M. Sansano }}{ }^{\mathrm{c}}$ \\ ${ }^{a}$ Department of Chemistry Institute for Advanced Studies in Basic Sciences (IASBS) P. O. \\ Box 45195-1159, Gavazang, Zanjan 45137-66731 (Iran).E-mail: gholinejad@iasbs.ac.ir \\ ${ }^{b}$ Research Center for Basic Sciences \& Modern Technologies (RBST) Institute for Advanced \\ Studies in Basic Sciences (IASBS) Zanjan 45137-66731 (Iran) \\ cDepartamento de Química Orgánica, Centro de Innovaciónen Química Avanzada \\ (ORFEO-CINQA), Instituto de Síntesis Orgánica (ISO), Facultad de Ciencias, Universidad \\ de Alicante, 03080 Alicante, Spain
}

Keywords: Human Hair, Catalyst, Reduction, Nitro, Amine

\begin{abstract}
Nowadays it is great challenge to use natural, cheap and biodegradable materials as catalyst in different organic reactions. In this work we use directly human hair dough as completely biodegradable, renewable, and available material in every locality for reduction of nitroarenes in aqueous media at $50{ }^{\circ} \mathrm{C}$. Using this new catalyst, structurally different aromatic nitro compounds, as well as heterocyclic ones are reduced to corresponding amines in high to excellent yields. The presented catalytic system is applicable for large scale reduction of nitroarenes.
\end{abstract}




\section{Introduction}

Reduction of nitro group has emerged as the most promising synthetic route to production of amine derivatives, which are essential intermediates in dyes, pigments,

pharmaceuticals and agrochemicals. ${ }^{1,2}$ Traditional approaches such as Béchamp reduction method, generates large amount of waste and requires mineral acids, which is not appropriate for acid sensitive functional groups. ${ }^{3}$ In recent years, an increasing attention has been paid to catalytic nitro reduction using transition metals such as $\mathrm{Cu},{ }^{4,5} \mathrm{Co},{ }^{6,7} \mathrm{Pd}^{8-11} \mathrm{Ni}^{12,13} \mathrm{Au}^{14-17}$ and $\mathrm{Zn}^{18,19}$. Along this line, due to the superior catalytic activity in bimetallic variants relative to their monometallic analogs, variety of bimetallic catalysts have been developed for organic reactions including the reduction of nitroarenes. ${ }^{20-26}$ However, it is still a great sustainable processes challenge to develop efficient, green and cheap catalysts for selective reduction of nitro group to amines.

Human hair is considered a minor excretory organ for trace elements such as $\mathrm{Fe}, \mathrm{Au}$, $\mathrm{Cu}, \mathrm{Mn}, \mathrm{Mg}$ and $\mathrm{Cr}, \mathrm{Co}, \mathrm{Zn}, \mathrm{Ni}$, etc. ${ }^{27-32}$ The content of trace elements in hair depend on different factors such geographic residence, environmental characteristics, age, 
sex, and color of hair. For example, red hair contains relatively high concentrations

of iron while white hair has low concentrations of manganese. ${ }^{33-38}$

In the past decade, due to environmental issues and efforts for development of sustainable chemistry, attention to the use of hair in various sciences has increased.

For instance, recently human hair is used as a support for stabilizing of different transition metal nanoparticles such as $\mathrm{Ni}^{39,40} \mathrm{Ru},{ }^{39-41} \mathrm{Au},{ }^{40,42} \mathrm{Ag},{ }^{41,42}$ and $\mathrm{Cu}^{40}$ resulting material have been applied as catalyst for various organic reactions. However, to the best of our knowledge, any report of using hair as a catalyst in any organic reaction has not been documented. Therefore, in continuation of our interest in development of abundant and naturally based catalysts, ${ }^{43-48}$ in this work for the first time we used human hair as catalyst for the reduction of structurally different nitroarenes in aqueous media under mild reaction conditions. 


\section{Experimental}

\subsection{General:}

Reactions were monitored by gas chromatography (GC) and thin layer chromatography (TLC) using Merck silica gel 60F254 glass plate with $0.25 \mathrm{~mm}$ thickness. ${ }^{1} \mathrm{H}$ NMR and ${ }^{13} \mathrm{C}$ NMR spectra were recorded at $400 \mathrm{MHz}$ and $100 \mathrm{MHz}$ respectively, on a Bruker Avance $\mathrm{HD}$ apparatus in DMSO- $d_{6}$ and $\mathrm{CDCl}_{3}$ and chemical shifts are given on the $\delta$-scale in ppm. The TEM and SEM images were captured with EOL JEM-2010 and JEOL JSM 840, respectively. XPS analyses were performed using a K-Alpha spectrometer. EDX results were obtained using a Carl Zeiss Sigma instrument.

\subsection{General procedure for the hair catalyzed reduction of nitroarenes:}

Initially, $2 \mathrm{~g}$ of human hair crushed into small pieces, washed with distilled water and then vacuum dried. Then, aqueous solution of $\mathrm{NaOH}\left(3.7 \mathrm{mmol}\right.$ in $\left.3 \mathrm{~mL} \mathrm{H}_{2} \mathrm{O}\right)$ and $1 \mathrm{~g}$ of the crushed human hair was added to the reaction tube and stirred at 50 ${ }^{\circ} \mathrm{C}$ until dissolving the hair. Afterward nitroarenes (0.4 mmol), $\mathrm{NaBH}_{4}(0.8 \mathrm{mmol}$, 
$30 \mathrm{mg})$ and $\mathrm{EtOH}(0.75 \mathrm{~mL})$ were added and the reaction mixture was stirred for appropriate reaction time at $50{ }^{\circ} \mathrm{C}$. Then, reaction mixture was washed with ethyl acetate $(3 \times 5 \mathrm{~mL})$ and the organic phase was separated. Further purification of amine product was performed by plate or column chromatography using hexane and ethyl acetate as eluents.

\section{Results and discussions}

Initially, structure of fresh human hair was studied with energy-dispersive X-ray (EDX), X-ray photoelectron spectroscopy (XPS), scanning electron microscopy (SEM) and transmission electron microscopy (TEM) analysis. EDX analysis of hair showed different elements such as $\mathrm{Na}, \mathrm{Ca}, \mathrm{C}, \mathrm{O}, \mathrm{N}, \mathrm{Al}, \mathrm{Si}, \mathrm{S}$, and $\mathrm{Ca}$ (Fig. 1). 


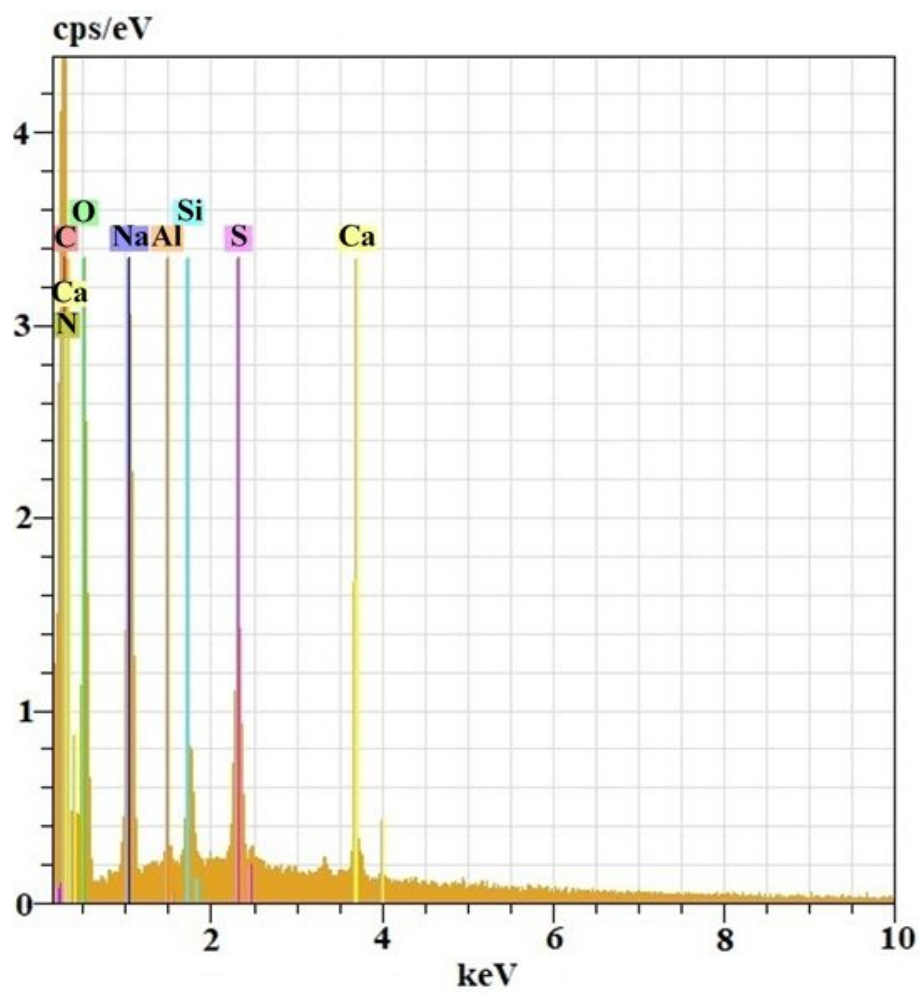

Fig. 1 EDX spectrum of human hair

The presence of $\mathrm{C}$ and $\mathrm{N}$ was further confirmed by XPS by showing peaks related to $\mathrm{C} 1 \mathrm{~s}$ at $284.7,285.5,286.4$, and $288.1 \mathrm{eV}$ are assigned to $\mathrm{C}-\mathrm{C}$ or $\mathrm{C}=\mathrm{C}, \mathrm{C}-\mathrm{N}, \mathrm{C}-\mathrm{O}$, and $\mathrm{C}=\mathrm{O}{ }^{49,50}$ and a peak at $400 \mathrm{eV}$ related to $\mathrm{N} 1 \mathrm{~s}^{51}$ (Fig. 2). 


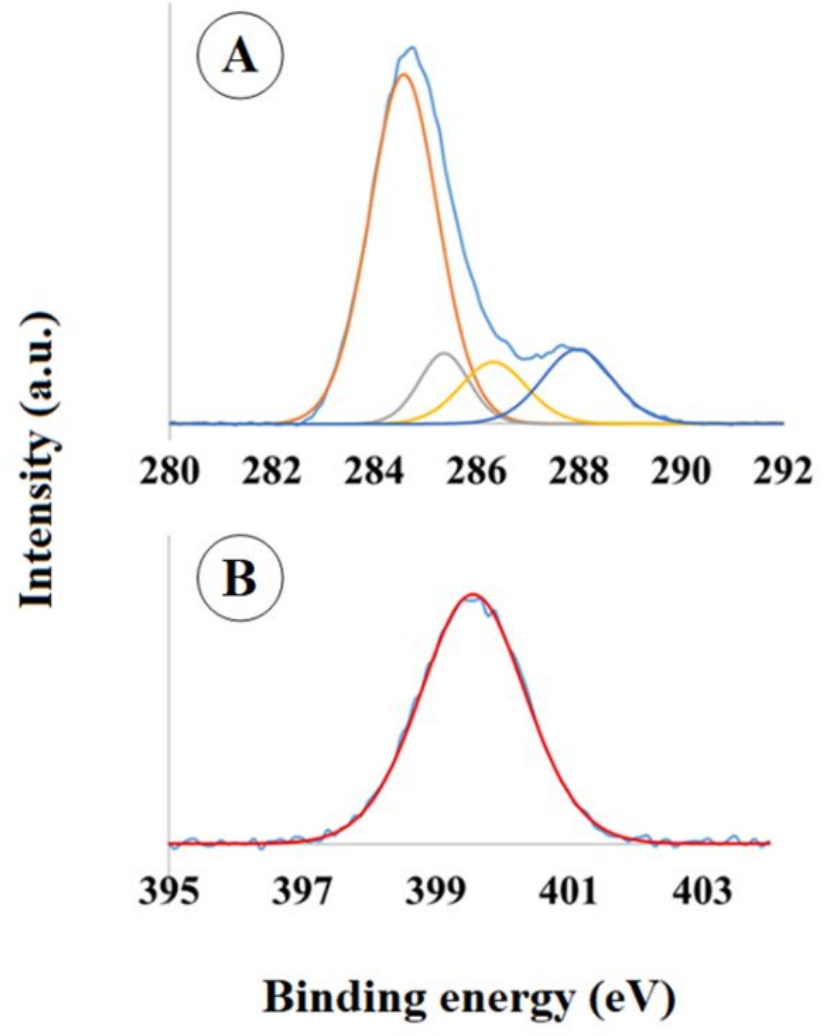

Fig. 2 XPS spectra of hair in A) C 1s and B) N 1s regions.

\begin{abstract}
Also, SEM of hair (after and before) of the treatment with alkaline solution was studied. SEM images of hair before treating with $\mathrm{NaOH}$ showed the presence of very uniform hair strand (Fig. 3). However, SEM images after treating with alkaline media exhibited ununiformed broken hair particles (Fig. 4). Also, TEM images of hair, after treating with alkaline media, showed the presence of hair layers (Fig. 1 ESI).
\end{abstract}




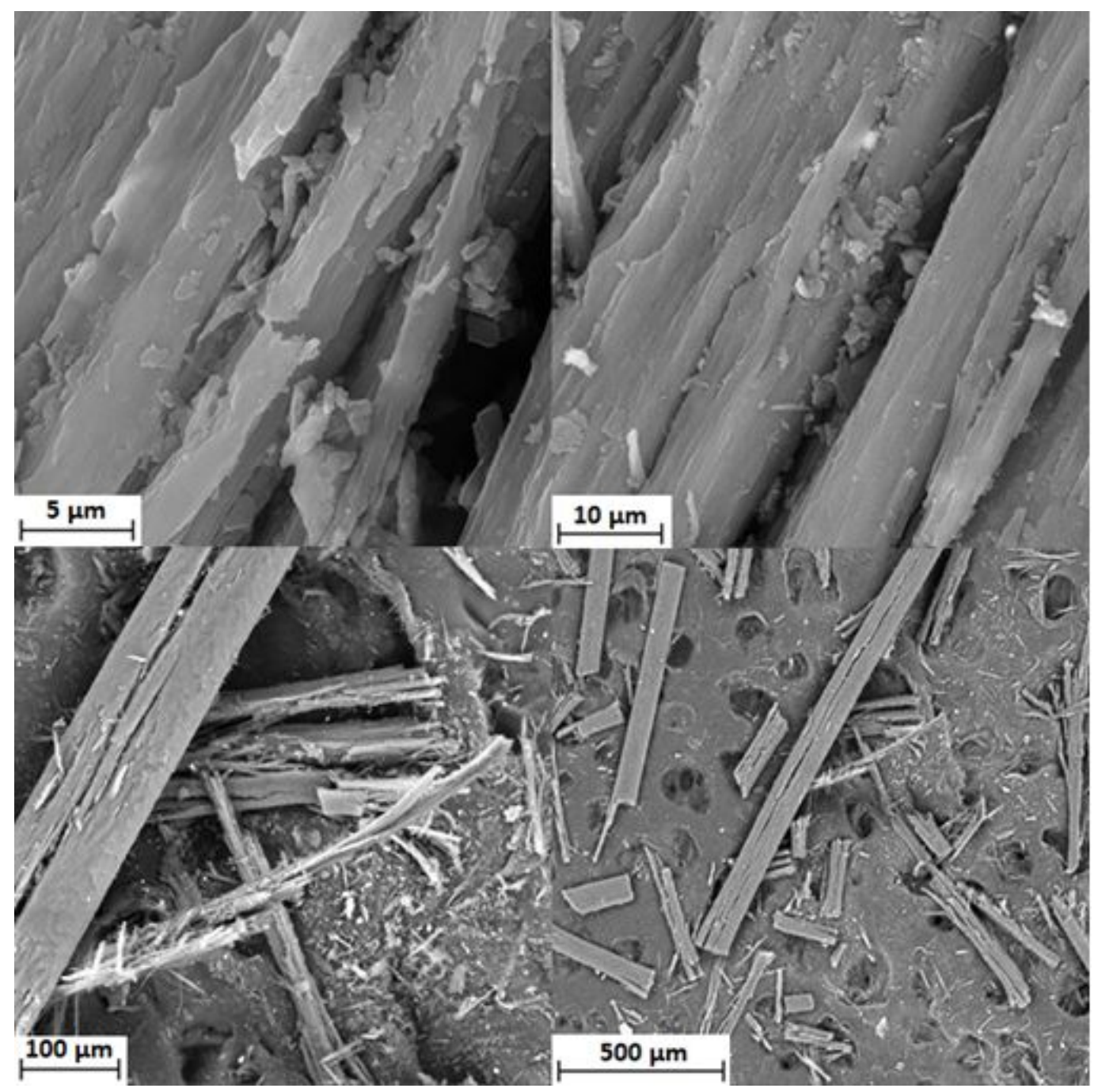

Fig. 3 SEM images of human hair before treating with $\mathrm{NaOH}$ solution 


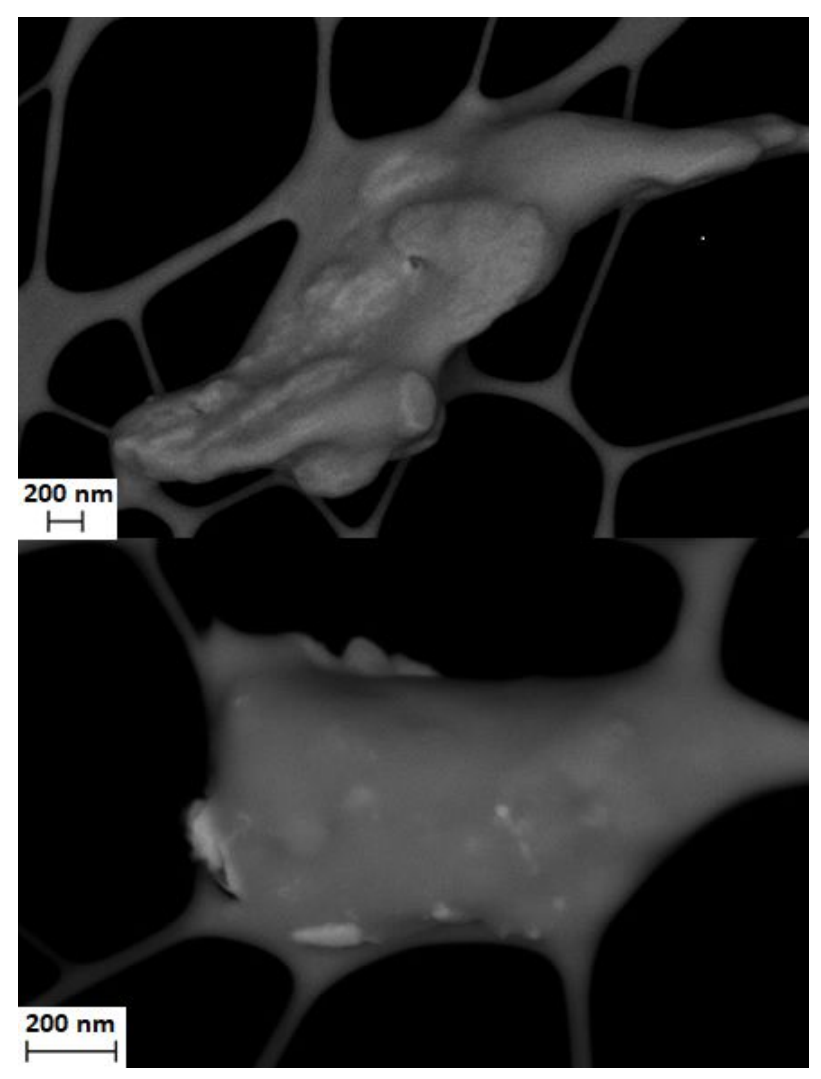

Fig. 4 SEM images of human hair after treating with $\mathrm{NaOH}$ solution

In order to study the catalytic activity of hair, reduction of 4-nitrotoluene (1a) was selected as benchmark reaction and the effects of human hair amount, solvent, temperature, and reducing agent on the yield of reaction were investigated.

Initially effect of different hair amounts and solvents were evaluated. The study of the reaction in solvents such as $\mathrm{EtOH}$, DMF, toluene, $\mathrm{CH}_{3} \mathrm{CN}$ in the presence of 0.4 g of human hair, $\mathrm{NaOH}(3.7 \mathrm{mmol})$ and $\mathrm{NaBH}_{4}(0.8 \mathrm{mmol})$ gave very poor yields during 6h (Table 1, entries 1-4). However, using solvents such as $\mathrm{H}_{2} \mathrm{O}$ and $\mathrm{H}_{2} \mathrm{O}$ : $\mathrm{EtOH}(1: 0.25)$ in the presence $\mathrm{NaOH}(3.7 \mathrm{mmol})$ afforded $17 \%$ and $25 \%$ yields, 
respectively (Table 1, entries 5 and 6). Selecting $\mathrm{H}_{2} \mathrm{O}: \mathrm{EtOH}(1: 0.25)$ as a solvent and increasing the hair amounts, yields were improved and $80 \%$ yield was achieved using $1 \mathrm{~g}$ of the hair (Table 1, entry 9). Results indicated that with increasing reaction times, yields were further improved and quantitative yield was achieved after $12 \mathrm{~h}$ (Table 1, entry 12). It should be noted that with decreasing $\mathrm{NaBH}_{4}$ amounts to 0.4 mmol, the yield of desired product was dropped to $67 \%$ (Table 1, entry 13). Also, the reactions in the absence of $\mathrm{NaBH}_{4}$ and also in the absence of hair failed to proceed (Table 1, entries 14-16). For possibility of metal leaching from hair, we washed hair with EDTA $(40 \mathrm{~mL}, 0.5 \mathrm{M})$ for $2 \mathrm{~h}$ and then used it for the reduction of 4-nitrotoluene under most optimized condition (Table 1, entry 12). Results indicted formation of desired product in $90 \%$ yield confirming lack of leaching of active catalysts in the presence of EDTA (Table 1, entry 17). Performing reaction using woman's brown hair gave also excellent yield (Table 1, entry 18). However, using dyed hair and natural white hair $20 \%$ and $46 \%$ yield was achieved, respectively (Table 1, entries 19 and 20). Finally, we tested cat's hair obtained from veterinary clinic in the reduction, but reaction did not proceed and starting material was intact (Table 1, entry 21). 
Table 1 Optimization of the reaction conditions for the reduction of 4-nitrotoluene (1a). ${ }^{\mathrm{a}}$

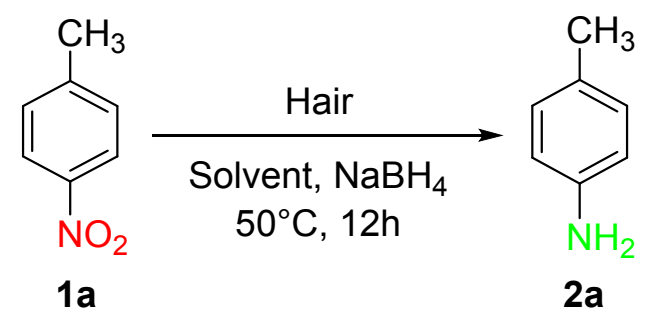

\begin{tabular}{|c|c|c|c|c|}
\hline Entry & Solvent & Time (h) & Hair (g) & Yield $(\%)^{b}$ \\
\hline 1 & $\mathrm{EtOH}$ & 6 & 0.4 & 5 \\
\hline 2 & DMF & 6 & 0.4 & $<2$ \\
\hline 3 & Toluene & 6 & 0.4 & $<1$ \\
\hline 4 & $\mathrm{CH}_{3} \mathrm{CN}$ & 6 & 0.4 & $<1$ \\
\hline 5 & $\mathrm{H}_{2} \mathrm{O}$ & 6 & 0.4 & 17 \\
\hline 6 & $\mathrm{H}_{2} \mathrm{O}: \mathrm{EtOH}$ & 6 & 0.4 & 25 \\
\hline 7 & $\mathrm{H}_{2} \mathrm{O}: \mathrm{EtOH}$ & 6 & 0.6 & 30 \\
\hline 8 & $\mathrm{H}_{2} \mathrm{O}: \mathrm{EtOH}$ & 6 & 0.8 & 55 \\
\hline 9 & $\mathrm{H}_{2} \mathrm{O}: \mathrm{EtOH}$ & 6 & 1 & 80 \\
\hline 10 & $\mathrm{H}_{2} \mathrm{O}: \mathrm{EtOH}$ & 7 & 1 & 85 \\
\hline 11 & $\mathrm{H}_{2} \mathrm{O}: \mathrm{EtOH}$ & 9 & 1 & 90 \\
\hline 12 & $\mathrm{H}_{2} \mathrm{O}: \mathrm{EtOH}$ & 12 & 1 & $>99$ \\
\hline 13 & $\mathrm{H}_{2} \mathrm{O}: \mathrm{EtOH}$ & 12 & 1 & $67^{c}$ \\
\hline 14 & $\mathrm{H}_{2} \mathrm{O}: \mathrm{EtOH}$ & 12 & - & $<1^{\mathrm{d}}$ \\
\hline
\end{tabular}




\begin{tabular}{ccccc}
\hline 15 & $\mathrm{H}_{2} \mathrm{O}:$ EtOH & 12 & 1 & $14^{\mathrm{e}}$ \\
16 & $\mathrm{H}_{2} \mathrm{O}:$ EtOH & 12 & - & $<1^{\mathrm{f}}$ \\
17 & $\mathrm{H}_{2} \mathrm{O}:$ EtOH & 12 & 1 & $90^{\mathrm{g}}$ \\
18 & $\mathrm{H}_{2} \mathrm{O}: \mathrm{EtOH}$ & 12 & 1 & $>99^{\mathrm{h}}$ \\
19 & $\mathrm{H}_{2} \mathrm{O}: \mathrm{EtOH}$ & 12 & 1 & $20^{\mathrm{i}}$ \\
20 & $\mathrm{H}_{2} \mathrm{O}: \mathrm{EtOH}$ & 12 & 1 & $46^{\mathrm{j}}$ \\
21 & $\mathrm{H}_{2} \mathrm{O}: \mathrm{EtOH}$ & 12 & 1 & $<1^{\mathrm{k}}$
\end{tabular}

\footnotetext{
a Reaction condition: human hair dissolved in solution of $\mathrm{NaOH}$ (3.7 $\mathrm{mmol}$ in $3 \mathrm{~mL}$ solvent), 4-nitrotoluene $(0.4 \mathrm{mmol})$ and $\mathrm{NaBH}_{4}(0.8 \mathrm{mmol})$.

$\mathrm{b}$ Yields determined by GC.

c Using $0.4 \mathrm{mmol}$ of $\mathrm{NaBH}_{4}$

${ }^{d}$ Reaction performed in the absence of hair and $\mathrm{NaOH}$

e Reaction performed in the absence of $\mathrm{NaBH}_{4}$

${ }^{\mathrm{f}}$ Reaction performed in the absence of hair and in the presence $3.7 \mathrm{mmol} \mathrm{NaOH}$ and $\mathrm{NaBH}_{4}(0.8 \mathrm{mmol})$.

${ }^{\mathrm{g}}$ Reaction performed using hair washed with EDTA

${ }^{\mathrm{h}}$ Reaction performed using woman's brown hair

${ }^{\mathrm{i}}$ Reaction performed using yellow dyed hair

${ }^{\mathrm{j}}$ Reaction performed using natural white hair of old men.

${ }^{\mathrm{k}}$ Reaction performed using cat's hair
}

In Table 2 it is demonstrated that the efficiency of $\mathrm{NaBH}_{4}$ is extremely high compared with other reducing agents tested such as formic acid, isopropyl alcohol, hydrazine, glycerol, and ascorbic acid. 
Table 2 Study of the efficiency of different reducing agents in the reduction of 4-nitrotoluene $(1 \mathbf{a}) .^{\mathrm{a}}$

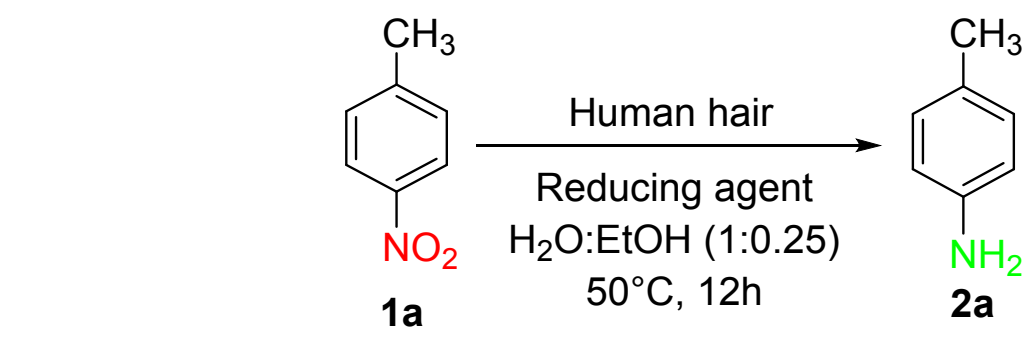

\begin{tabular}{ccc}
\hline Entry & Reductant & Yield (\%) $^{\mathbf{b}}$ \\
\hline 1 & Formic acid & 20 \\
2 & Isopropyl Alcohol & 23 \\
3 & Hydrazine & 22 \\
4 & Glycerol & 18 \\
5 & Ascorbic acid & 28 \\
6 & Sodium borohydride & $>99$
\end{tabular}

${ }^{a}$ Reaction condition: human hair $(1 \mathrm{~g})$ dissolved in aqueous solution of $\mathrm{NaOH}\left(3.7 \mathrm{mmol}\right.$ in $\left.3 \mathrm{~mL} \mathrm{H}_{2} \mathrm{O}\right)$, 4nitrotoluene $(0.4 \mathrm{mmol})$, EtOH $(0.75 \mathrm{~mL})$ and reducing agent $(0.8 \mathrm{mmol})$ at $50{ }^{\circ} \mathrm{C}$.

b Yields determined by GC.

Having optimized conditions in hand (Table 1, entry 12), we investigated the reduction reaction of variety of nitro compounds 1 (Scheme 1). Results indicated that reduction of aromatic nitro compounds bearing electron withdrawing groups in the $1 \mathbf{c}-1 \mathbf{e}, \mathbf{1 h}, \mathbf{1 m}-\mathbf{1 0}$ and $\mathbf{1 p}$, as well as electron donating groups in the $1 \mathbf{a}, \mathbf{1 b}$, 
1f, $\mathbf{1 h}, \mathbf{1} \mathbf{j}-\mathbf{1 l}$ and $\mathbf{1 q}-\mathbf{1 s}$ were proceeded very well and $80-99 \%$ yields were obtained (Table 3 ). It should be noted that in the case of nitro aromatic compounds having formyl group in the $\mathbf{1} \mathbf{m}-\mathbf{1 0}$, both carbonyl and nitro groups were reduced to alcohol and amine, respectively. while in the case methyl 4-nitrobenzoate (1p), nitro group was reduced to amine without reduction of ester group.

Scheme 1 Reduction of structurally different nitroarenes 1 to the amines counterpart 2 using human hair as a catalyst.

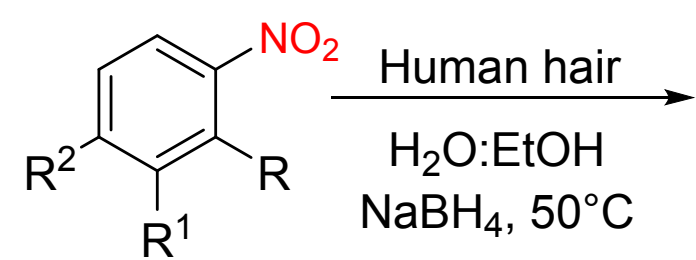

1

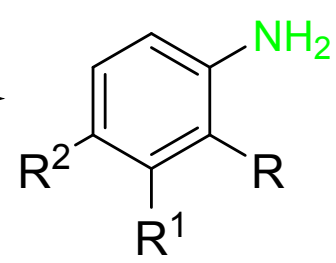

2
a $R=H, R^{1}=H, R^{2}=M e$
a $\mathrm{R}=\mathrm{H}, \mathrm{R}^{1}=\mathrm{H}, \mathrm{R}^{2}=\mathrm{Me}$
b $\mathrm{R}=\mathrm{H}, \mathrm{R}^{1}=\mathrm{H}, \mathrm{R}^{2}=\mathrm{OMe}$
b $\mathrm{R}=\mathrm{H}, \mathrm{R}^{1}=\mathrm{H}, \mathrm{R}^{2}=\mathrm{OMe}$
c $R=H, R^{1}=H, R^{2}=\mathrm{Cl}$
c $\mathrm{R}=\mathrm{H}, \mathrm{R}^{1}=\mathrm{H}, \mathrm{R}^{2}=\mathrm{Cl}$
d $\mathrm{R}=\mathrm{H}, \mathrm{R}^{1}=\mathrm{H}, \mathrm{R}^{2}=\mathrm{Br}$
d $\mathrm{R}=\mathrm{H}, \mathrm{R}^{1}=\mathrm{H}, \mathrm{R}^{2}=\mathrm{Br}$
e $\mathrm{R}=\mathrm{H}, \mathrm{R}^{1}=\mathrm{NO}_{2}, \mathrm{R}^{2}=\mathrm{Cl}$
e $\mathrm{R}=\mathrm{H}, \mathrm{R}^{1}=\mathrm{NH}_{2}, \mathrm{R}^{2}=\mathrm{Cl}$
f $\mathrm{R}=\mathrm{H}, \mathrm{R}^{1}=\mathrm{NH}_{2}, \mathrm{R}^{2}=\mathrm{H}$
f $\mathrm{R}=\mathrm{H}, \mathrm{R}^{1}=\mathrm{NH}_{2}, \mathrm{R}^{2}=\mathrm{H}$
g $\mathrm{R}=\mathrm{NO}_{2}, \mathrm{R}^{1}=\mathrm{H}, \mathrm{R}^{2}=\mathrm{H}$
g R $=\mathrm{NH}_{2}, \mathrm{R}^{1}=\mathrm{H}, \mathrm{R}^{2}=\mathrm{H}$
h R $=\mathrm{NO}_{2}, \mathrm{R}^{1}=\mathrm{H}, \mathrm{R}^{2}=\mathrm{Cl}$
h $\mathrm{R}=\mathrm{NH}_{2}, \mathrm{R}^{1}=\mathrm{H}, \mathrm{R}^{2}=\mathrm{Cl}$
i $\mathrm{R}=\mathrm{H}, \mathrm{R}^{1}=\mathrm{H}, \mathrm{R}^{2}=\mathrm{H}$
i $\mathrm{R}=\mathrm{H}, \mathrm{R}^{1}=\mathrm{H}, \mathrm{R}^{2}=\mathrm{H}$
j R= $\mathrm{CH}_{2} \mathrm{OH}, \mathrm{R}^{1}=\mathrm{H}, \mathrm{R}^{2}=\mathrm{H}$
j $\mathrm{R}=\mathrm{CH}_{2} \mathrm{OH}, \mathrm{R}^{1}=\mathrm{H}, \mathrm{R}^{2}=\mathrm{H}$
k R $=\mathrm{H}, \mathrm{R}^{1}=\mathrm{CH}_{2} \mathrm{OH}, \mathrm{R}^{2}=\mathrm{H}$
k R $=\mathrm{H}, \mathrm{R}^{1}=\mathrm{CH}_{2} \mathrm{OH}, \mathrm{R}^{2}=\mathrm{H}$
I R $=\mathrm{H}, \mathrm{R}^{1}=\mathrm{H}, \mathrm{R}^{2}=\mathrm{CH}_{2} \mathrm{OH}$
$\mathrm{I} R=\mathrm{H}, \mathrm{R}^{1}=\mathrm{H}, \mathrm{R}^{2}=\mathrm{CH}_{2} \mathrm{OH}$
m R= CHO, $R^{1}=H, R^{2}=H$
m R $=\mathrm{CH}_{2} \mathrm{OH}, \mathrm{R}^{1}=\mathrm{H}, \mathrm{R}^{2}=\mathrm{H}$
n $\mathrm{R}=\mathrm{H}, \mathrm{R}^{1}=\mathrm{CHO}, \mathrm{R}^{2}=\mathrm{H}$
n $\mathrm{R}=\mathrm{H}, \mathrm{R}^{1}=\mathrm{CH}_{2} \mathrm{OH}, \mathrm{R}^{2}=\mathrm{H}$
o $\mathrm{R}=\mathrm{H}, \mathrm{R}^{1}=\mathrm{H}, \mathrm{R}^{2}=\mathrm{CHO}$
o $\mathrm{R}=\mathrm{H}, \mathrm{R}^{1}=\mathrm{H}, \mathrm{R}^{2}=\mathrm{CH}_{2} \mathrm{OH}$ 


$$
\begin{aligned}
& \text { p } \mathrm{R}=\mathrm{H}, \mathrm{R}^{1}=\mathrm{H}, \mathrm{R}^{2}=\mathrm{CO}_{2} \mathrm{Me} \quad \text { p } \mathrm{R}=\mathrm{H}, \mathrm{R}^{1}=\mathrm{H}, \mathrm{R}^{2}=\mathrm{CO}_{2} \mathrm{Me} \\
& \text { q } \mathrm{R}=\mathrm{OH}, \mathrm{R}^{1}=\mathrm{H}, \mathrm{R}^{2}=\mathrm{H} \quad \text { q } \mathrm{R}=\mathrm{OH}, \mathrm{R}^{1}=\mathrm{H}, \mathrm{R}^{2}=\mathrm{H} \\
& r \mathrm{R}=\mathrm{H}, \mathrm{R}^{1}=\mathrm{OH}, \mathrm{R}^{2}=\mathrm{H} \quad \mathbf{R}=\mathrm{H}, \mathrm{R}^{1}=\mathrm{OH}, \mathrm{R}^{2}=\mathrm{H} \\
& \text { s } \mathrm{R}=\mathrm{H}, \mathrm{R}^{1}=\mathrm{H}, \mathrm{R}^{2}=\mathrm{OH} \quad \mathbf{s}=\mathrm{H}, \mathrm{R}^{1}=\mathrm{H}, \mathrm{R}^{2}=\mathrm{OH} \\
& \text { t } \mathrm{R}=\mathrm{H}, \mathrm{R}^{1}=2,5 \text { dichloropyrimidin-4- } \quad \mathbf{t} \mathrm{R}=\mathrm{H}, \mathrm{R}^{1}=2,5 \text { dichloropyrimidin- } \\
& \text { amino, } R^{2}=\mathrm{H} \quad \text { 4-amino, } \mathrm{R}^{2}=\mathrm{H} \\
& \text { u } R=H, R^{1}=H, R^{2}=P h \quad \text { u } R=H, R^{1}=H, R^{2}=P h
\end{aligned}
$$

Table 3 Reduction of structurally different nitroarenes $\mathbf{1}$ to the amines counterpart $\mathbf{2}$ using human hair as a catalyst, according to scheme $1 .^{\mathrm{a}}$

\begin{tabular}{ccccc}
\hline Entry & Ar-NO & Time (h) & Product & Yield (\%) $^{\mathbf{b}}$ \\
\hline 1 & $\mathbf{1 a}$ & 12 & $\mathbf{2 a}$ & $99^{\mathrm{c}}$ \\
2 & $\mathbf{1 b}$ & 12 & $\mathbf{2 b}$ & $98^{\mathrm{c}}$ \\
3 & $\mathbf{1 c}$ & 10 & $\mathbf{2 c}$ & $98^{\mathrm{c}}$ \\
4 & $\mathbf{1 d}$ & 10 & $\mathbf{2 d}$ & $80^{\mathrm{c}}$ \\
5 & $\mathbf{1 e}$ & 10 & $\mathbf{2 e}$ & $90^{\mathrm{d}, \mathrm{e}}$ \\
6 & $\mathbf{1 f}$ & 9 & $\mathbf{2 f}$ & $87^{\mathrm{c}, \mathrm{e}}$ \\
7 & $\mathbf{1 g}$ & 10 & $\mathbf{2 g}$ & $89^{\mathrm{d}, \mathrm{e}}$ \\
8 & $\mathbf{1 h}$ & 9 & $\mathbf{2 h}$ & $90^{\mathrm{c}, \mathrm{e}}$ \\
9 & $\mathbf{1 i}$ & 8 & $\mathbf{2 i}$ & $99^{\mathrm{c}}$ \\
10 & $\mathbf{1 j}$ & 11 & $\mathbf{2 j}$ & $96^{\mathrm{c}}$ \\
11 & $\mathbf{1 k}$ & 12 & $\mathbf{2 k}$ & $94^{\mathrm{c}}$ \\
12 & $\mathbf{1 1}$ & 10 & $\mathbf{2 l}$ & $90^{\mathrm{c}, \mathrm{e}}$ \\
13 & $\mathbf{1 m}$ & 11 & $\mathbf{2 m}$ & $97^{\mathrm{d}}$ \\
\hline
\end{tabular}




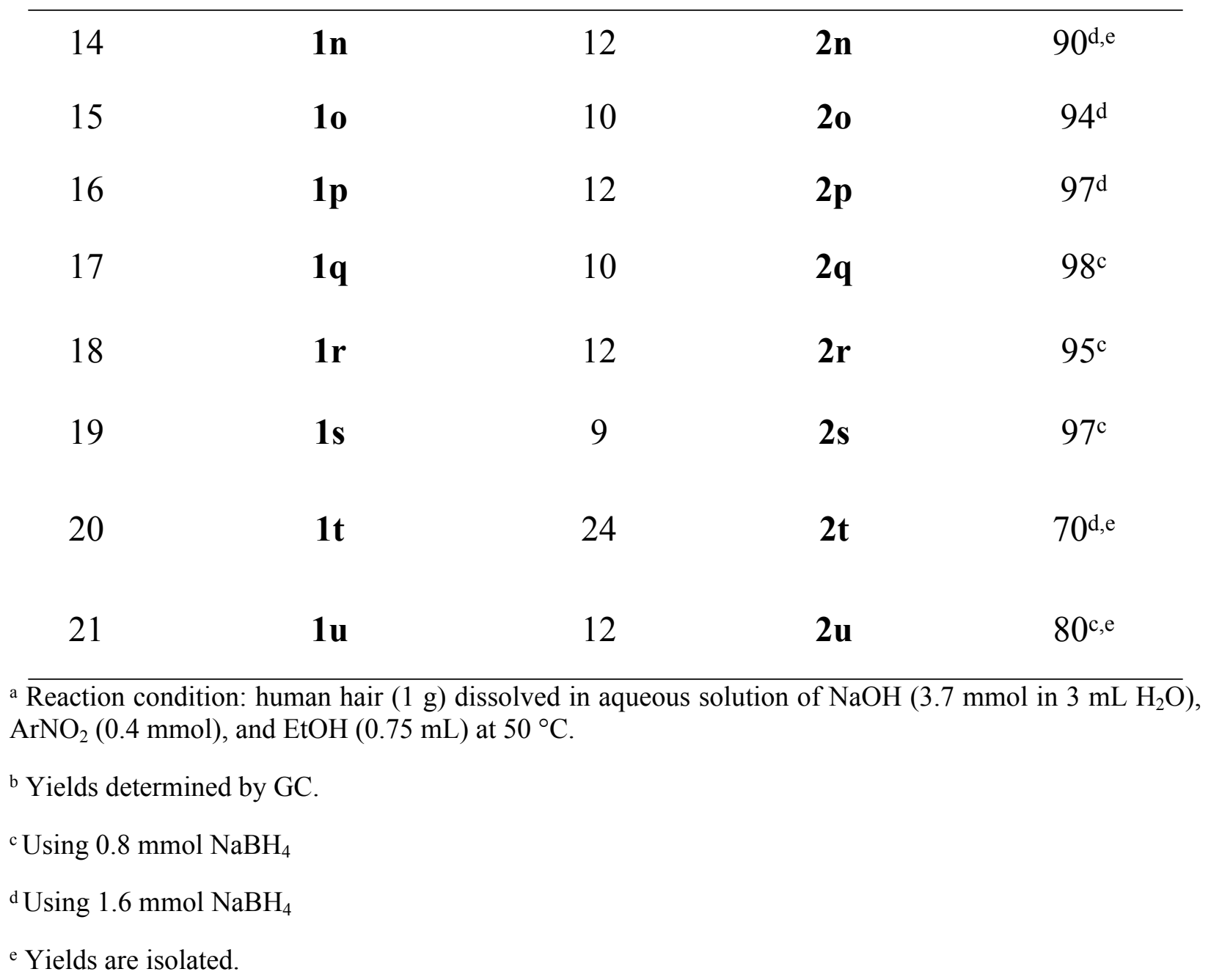

In order to show the features of the reaction for large-scale operation, we have scaled-up the reaction of 4-nitrotoluene (1a) to the $10 \mathrm{mmol}$, under optimized reaction conditions. Results indicated that reaction proceeded well and desired amine was obtained in $85 \%$ isolated yield (scheme 2). 


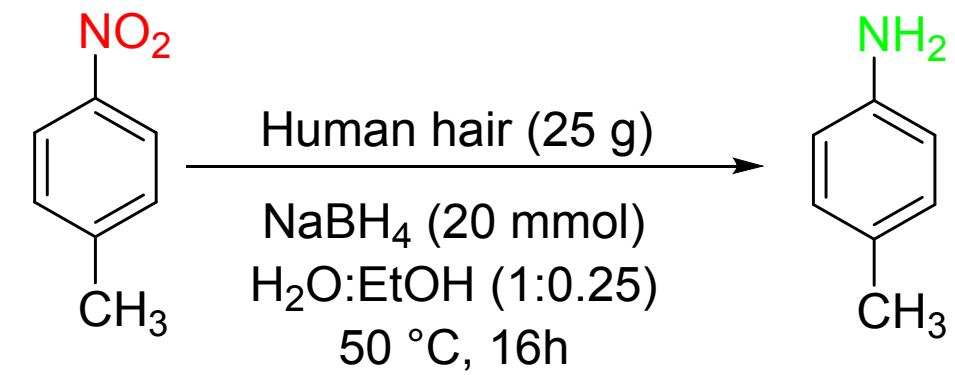

$10 \mathrm{mmol}$

$85 \%$

$1 \mathrm{a}$

$2 a$

Scheme 2 Reduction of 4-nitrotoluene (1a) in a large scale.

\section{Conclusions}

In conclusion, in this study we have presented a novel protocol in which human hair has been used as a catalyst for the important nitroarene reduction in aqueous media. By this method the structurally diverse nitroaromatic compounds have been reduced to corresponding amines in high to excellent yields under mild reaction condition. This method is applicable for large scale operation which is of interest for industrial purposes. 


\section{Acknowledgments}

The authors are grateful to Institute for Advanced Studies in Basic Sciences (IASBS)

Research Council, Iran National Science Foundation (INSF-Grant number of 97021804) and the University of Alicante. We also thankful for financial support to the Spanish Ministerio de Econom_a y Competitividad (MINECO; projects CTQ2013-43446-P and CTQ2014-51912-REDC), the Spanish Ministerio de Econom_a, Industria y Competitividad, Agencia Estatal de Investigaci_n (AEI) and Fondo Europeo de Desarrollo Regional (FEDER, EU; projects CTQ2016-76782-P and CTQ2016-81797-REDC), the Generalitat Valenciana (PROMETEOII/2014/017) and the University of Alicante. Special 


\section{References}

(1) Orlandi, M.; Brenna, D.; Harms, R.; Jost, S. \& Benaglia, M. Org. Process Res. Dev. 2018, 22, 430-445. doi: 10.1021/acs.oprd.6b00205

(2) Goksu, H.; Sert, H.; Kilbas, B.; \& Sen, F. Curr. Org. Chem. 2017, 21, 794-820.

(3) Béchamp, A. Ann. Chim. Phys. 1854, 42, 186.

(4) Deka, P.; Deka, R. C.; Bharali, P. New J. Chem. 2014, 38, 1789-1793. doi: 10.1039/C3NJ01589K

(5) Bai, Y.; Wang, Q.; Du, C.; Bu, T.; Liu, Y.; Sun, X.; Wang, L. J. Colloid Interf.

Sci. 2019, 553, 768-777. doi: 10.1016/j.inoche.2019.107588

(6) Sahoo, B.; Formenti, D.; Topf, C.; Bachmann, S.; Scalone, M.; Junge, K. \& Beller, M. ChemSusChem 2017, 10, 3035-3039. doi: 10.1002/cssc.201700796

(7) Shultz, L. R.; McCullough, B.; Newsome, W. J.; Ali, H.; Shaw, T. E.; Davis, K. O.; Jurca, T. Molecules, 2020, 25, 89. doi: 10.3390/molecules25010089

(8) Zhang, K.; Hong, K.; Suh, J. M.; Lee, T. H.; Kwon, O.; Shokouhimehr, M. \& Jang, H. W. Res. Chem. Intermed. 2019, 45, 599-611. doi: 10.1007/s11164-018$3621-8$ 
(9) Uberman, P. M.; García, C. S.; Rodríguez, J. R. \& Martín, S. E. Green Chem. 2017, 19, 739-748. doi: 10.1039/C6GC02710E

(10) Gholinejad, M.; Rasouli, Z.; Najera, C.; Sansano, J. M. ChemPlusChem 2019, 84, 1122-1129. doi: 10.1002/cplu.201900377

(11) Doherty, S.; Knight, J. G.; Backhouse, T.; Bradford, A.; Saunders, F.; Bourne, R. A.; Lovelock, K. Catal. Sci. Technol. 2018, 1454-1467. doi: 10.1039/C7CY02557B

(12) Yang, Y.; Ren, Y.; Sun, C. \& Hao, S. Green Chem. 2014, 16, 2273-2280. doi: 10.1039/C3GC42121J

(13) Romanazzi, G.; Fiore, A. M.; Mali, M.; Rizzuti, A.; Leonelli, C.; Nacci, A.; Mastrorilli, P. \& Dell'Anna, M. M. Mol. Catal. 2018, 446, 31-38. doi: 10.1016/j.mcat.2017.12.015

(14) Pourjavadi, P. A.; Keshavarzi, N.; Moghaddam, F. M. \& Hosseini, S. H. ChemistrySelect 2018, 3, 2716-2722. doi: 10.1002/slct.201702798

(15) Bhosale, M. A.; Chenna, D. R. \& Bhanage, B. M. ChemistrySelect 2017, 2, 1225-1231. doi: 10.1002/slct.201601851

(16) Fountoulaki, S.; Daikopoulou, V.; Gkizis, P. L.; Tamiolakis, I.; Armatas, G. S.; Lykakis, I. N. ACS Catalysis 2014, 4, 3504-3511. doi: 10.1021/cs500379u 
(17) Gholinejad, M.; Dasvarz, N.; Shojafar, M.; Sansano, J. M. Inorg. Chim. Acta 2019, 495, 118965. doi: 10.1002/cplu.201900377

(18) Sharma, U.; Kumar, N.; Verma, P. K.; Kumar, V. \& Singh, B. Green Chem. 2012, 14, 2289-2293. doi: 10.1039/C2GC35452G

(19) Toh, H. S.; Ambrosi, A.; Pumera, M. Catal. Sci. Technol. 2013, 3, 123-127. doi: 10.1039/C2CY20253K

(20) Huang, L.; Lv, Y.; Wu, S.; Liu, P.; Xiong, W.; Hao, F. \& Luo, H. A. Appl. Catal. A Gen. 2019, 577, 76-85. doi: 10.1016/j.apcata.2019.03.017

(21) Xu, X.; Li, H.; Xie, H.; Ma, Y.; Chen, T. \& Wang, J. J. Mater. Res. 2017, 32, 1777-1786. doi: 10.1557/jmr.2017.148

(22) Obraztsova, I. I.; Eremenko, N. K.; Yu Simenyuk, G.; Eremenko, A. N. \& Tryasunov, B. G. Solid Fuel Chem. 2012, 46, 364-367. doi: $10.3103 / \mathrm{S} 0361521912060109$

(23) Keshipour, S. \& Mirmasoudi, S. S. Advances in Environmental Technology 2017, 3, 59-65. doi: 10.22104/AET.2017.501

(24) Ghosh, S. K.; Mandal, M.; Kundu, S.; Nath, S. \& Pal, T. Appl. Catal. A Gen. 2004, 268, 61-66. doi: 10.1016/j.apcata.2004.03.017 
(25) Silva, T. R.; De Oliveira, D. C.; Pal, T. \& Domingos, J. B. New J. Chem. 2019, 43, 7083-7092. doi: 10.1039/C9NJ00285E

(26) Keshipour, S. \& Kamran, A. Iran. J. Chem. Chem. Eng. 2018, 37, 23-31.

(27) DiPietro, E. S.; Phillips, D. L.; Paschal, D. C. \& Neese, J. W. Biol. Trace Elem. Res. 1989, 22, 83. doi: 10.1007/BF02917419

(28) Kratzer, K.; Beneš, P.; Spěváčková, V.; Kolihová, D. \& Žilková, J. J. Anal. At. Spectrom. 1994, 9, 303-306. doi: 10.1039/JA9940900303

(29) Batzevich, V. A. Sci. Total Environ. 1995, 164, 89-98. doi: 10.1016/00489697(95)91020-4

(30) Dombovári, J. \& Papp, L. Microchem. J. 1998, 59, 187-193. doi: 10.1006/mchj.1998.1573

(31) Puchyr, R. F.; Bass, D. A.; Gajewski, R.; Calvin, M.; Marquardt, W.; Urek, K.; Druyan, M.E. \& Quig, D. Biol. Trace Elem. Res. 1998, 62, 167-182. doi: 10.1007/BF02783969

(32) Chojnacka, K.; Górecka, H.; Chojnacki, A. \& Górecki, H. Environ. Toxicol. Pharmacol. 2005, 20, 368-374. doi: 10.1016/j.etap.2005.03.004 
(33) Ward, N. I.; Spyrou, N. M. \& Damyanova, A. A. J. Radioanal. Nucl. Chem. 1987, 114, 125-135. doi: 10.1007/BF02048883

(34) Luo, R.; Zhuo, X. \& Ma, D. Ecotoxicol. Environ. Saf. 2014, 104, 215-219. doi: 10.1016/j.ecoenv.2014.03.006

(35) Chojnacka, K.; Górecka, H. \& Górecki, H. Sci. Total Environ. 2006, 366, 612620. doi: $10.1016 /$ j.scitotenv.2005.12.005

(36) Zaichick, S. \& Zaichick, V. Biol. Trace Elem. Res. 2009, 134, 41-54. doi: $10.1007 / \mathrm{s} 12011-009-8456-0$

(37) Zaichick, S. \& Zaichick, V. Int. J. Environ. Health 2011, 5, 106-124. doi: 10.1504/IJEnvH.2011.03986

(38) Skalnaya, M. G.; Tinkov, A. A.; Demidov, V. A.; Serebryansky, E. P.; Nikonorov, A. A. \& Skalny, A. V. J. Elem. 2016, 21, 211-220. doi: 10.5601.jelem.2015.20.3.924

(39) Gopiraman, M. \& Chung, I. M. Korean J. Chem. Eng. 2017, 34, 2169-2179. doi: 10.1007/s11814-017-0113-2

(40) Gopiraman, M.; Deng, D.; Zhang, K. Q.; Chung, I. M.; Karvembu, R. \& Kim, I. S. Ind. Eng. Chem. Res. 2017, 56, 1926-1939. doi: 10.1021/acs.iecr.6b04209 
(41) Gopiraman, M.; Saravanamoorthy, S. \& Chung, I. M. Res. Chem. Intermed. 2017, 43, 5601-5614. doi: 10.1007/s11164-017-2950-3

(42) Deng, D.; Gopiraman, M.; Kim, S. H.; Chung, I. M. \& Kim, I. S. ACS Sustain. Chem. Eng. 2016, 4, 5409-5414. doi: 10.1021/acssuschemeng.6b01689

(43) Gholinejad, M. \& Jeddi, N. ACS Sustainable Chem. Eng. 2014, 2, 2658-2665. doi: $10.1021 / \mathrm{sc} 500395 \mathrm{~b}$

(44) Gholinejad, M.; Bonyasi, R.; Najera, C.; Saadati, F.; Bahrami, M. \& Dasvarz, N. ChemPlusChem 2018, 83, 431-438. doi: 10.1002/cplu.201800162

(45) Gholinejad, M.; Afrasi, M. \& Najera, C. Appl. Organomet. Chem. 2019, 33, e4760. doi: 10.1002/aoc.4760

(46) Gholinejad, M.; Oftadeh, E. \& Sansano, J. M. ChemistrySelect 2019, 4, 31513160. doi: 10.1002/slct.201803599

(47) Lipshutz, B. H.; Gholinejad, M.; Oftadeh, E.; Shojafar, M. \& Sansano, J. M. ChemSusChem 2019, doi:10.1002/cssc.201901535

(48) Ghaderi, A.; Gholinejad, M. \& Firouzabadi, H. Curr. Org. Chem. 2016, 20, $327-348$. 
(49) Tu, Q.; Pang, L.; Chen, Y.; Zhang, Y.; Zhang, R.; Lu, B. \& Wang, J. Analyst, 2014, 139, 105-115. doi: 10.1039/C3AN01796F

(50) Gholinejad, M.; Zareh, F. \& Nájera, C. Appl. Organomet. Chem. 2018, 32, e3984. doi: 10.1002/aoc.3984

(51) Gholinejad, M.; Afrasi, M.; Nikfarjam, N. \& Nájera, C. Appl. Catal. A Gen. 2018, 563, 185-195. doi: 10.1016/j.apcata.2018.07.009. 


\section{List of Tables and Figures captions}

Fig. 1. EDX spectrum of human hair.

Fig. 2. XPS spectra of hair in A) C 1s and B) $\mathrm{N} 1 \mathrm{~s}$ regions.

Fig. 3. SEM images of human hair before treating with $\mathrm{NaOH}$ solution

Fig. 4. SEM images of human hair after treating with $\mathrm{NaOH}$ solution

Table 1. Optimization of the reaction conditions for the reduction of 4-nitrotoluene (1a).

Table 2. Study of the efficiency of different reducing agents in the reduction of 4-nitrotoluene (1a).

Scheme 1. Reduction of structurally different nitroarenes $\mathbf{1}$ to the amines counterpart $\mathbf{2}$ using human hair as a catalyst.

Table 3. Reduction of structurally different nitroarenes $\mathbf{1}$ to the amines counterpart $\mathbf{2}$ using human hair as a catalyst, according to scheme 1 .

Scheme 2. Reduction of 4-nitrotoluene (1a) in a large scale. 